\title{
Prerequisites for the Modelling and Analysis of a Product Development Process Using Network Theory
}

\author{
Abdo Chahin, Julian Hoffmeister, Kristin Paetzold and Vahid Salehi
}

\begin{abstract}
Network models have already been used with the intent to gain additional information about the structure of product development processes (PDP). These are supposed to map the flow of information and data as well as to provide a deeper understanding of the company's procedures. Process networks commonly represent dependencies of tasks and/or social contacts. Treating tasks as nodes in a network allows for a comparison of their position within the process. This way, it is possible to characterize certain actions according to their network attributes. In order to fully describe a PDP, it is, however, necessary to include other influencing factors as well. For example, there are only few approaches examining the impact of quality and progress on the process artefacts (such as CAD-files). The goal of this paper is to clarify what information is necessary to precisely describe a PDP in a network model. This covers a statement about the level of detail, general structure and dynamic of the networks.
\end{abstract}

\footnotetext{
A. Chahin $(\bowtie) \cdot$ K. Paetzold

Institute of Technical Product Development, Universität der Bundeswehr München, Neubiberg, Germany

e-mail: abdo.chahin@unibw.de

J. Hoffmeister · V. Salehi

Department of Applied Sciences and Mechatronics,

University of Applied Sciences Munich, Munich, Germany

G. Fanmuy et al. (eds.), Complex Systems Design \& Management,

DOI 10.1007/978-3-319-49103-5_20
} 